\title{
Android based Automated Irrigation System using Raspberry Pi
}

\author{
Suprabha Jadhav ${ }^{1}$, Shailesh Hambarde ${ }^{2}$ \\ ${ }^{1}$ Savitribai Phule Pune University, JSPM's JSCOE, Handewadi Road, Hadapsar, Pune-28, India
}

\begin{abstract}
Nowadays, adopting an optimized irrigation system has become a necessity due to the lack of the world water resource. The system has a distributed wireless network of soil-moisture and temperature sensors. This project focuses on a smart irrigation system which is cost effective. As the technology is growing and changing rapidly, Wireless sensing Network (WSN) helps to upgrade the technology where automation is playing important role in human life. Automation allows us to control various appliances automatically. DC motor based vehicle is designed for irrigation purpose. The objectives of this paper were to control the water supply to each plant automatically depending on values of temperature and soil moisture sensors. Mechanism is done such that soil moisture sensor electrodes are inserted in front of each soil. It also monitors the plant growth using various parameters like height and width. Android app.
\end{abstract}

Keywords: Android App, Automation, Raspberry Pi, Sensors.

\section{Introduction}

In China, water resource is in severe shortage and agricultural water consumption accounts for about $80 \%$ of the total water consumption. Because of out-dated agriculture irrigation method, the coefficient of irrigation water for effective utilization is only $0.3-0.4$ or so. One important way to improve the utilization rate of irrigation water is to implement precise controlled information on the basis of water crops demand data.

In the modern drip irrigation systems, the most significant advantage is that water is supplied near the root zone of the plants drip by drip due to which a large quantity of water is saved. At the present era, the farmers have been using irrigation technique in India through the manual control in which the farmers irrigate the land from time to time. This process sometimes consumes more water.

Automatic irrigation scheduling consistently has shown to be valuable in water use efficiency with respect to manual irrigation based on direct soil water measurements. The aim of the implementation is to demonstrate that the automatic irrigation can be used to reduce water use. The implementation is a automated irrigation system that consists of a distributed wireless network of soil moisture and temperature sensor.

There are different types of method for irrigating farm field for different types crop field. Basically Indian farmer use these three methods channel system, sprinkler system, drip system. Channel system is a traditional method of irrigation. But a smart irrigation system is a new technology to irrigating farm field automatically.

Drip irrigation is one of the methods of irrigation that saves water and fertilizer. In drip irrigation method, water drips slowly to the roots of the plants either onto the soil surface or directly onto the root zone through a network of valves, pipes, tubing and emitters. The process is completed in narrow tubes so that water is given directly to the root of the plant. Drip irrigation is also termed as localized irrigation or micro irrigation.

Main purpose of this project is to save the water and to increase the production of crops by monitoring the growth of plants. To substitute the flooding method/manual method of giving water to each plant by irrigation system. To design irrigation by drip that would be economical and efficient as water management has a crucial importance. To maximize utilization of available water. The conventional drip irrigation system is fully controlled and monitored by the farmer. This project presents a fully automated drip irrigation system which is controlled and monitored by using Raspberry Pi.

\subsection{Background}

Irrigation refers to the process of supply of water through artificial means such as pipes, ditches, sprinklers, etc. The irrigation system helps the farmers to have less dependency on rain-water for the purpose of agriculture. The necessity and importance of irrigation is highlighted below in points:

1) Variety of Climate: Indian climate and weather conditions experiences a varied range of climate. There is extreme heat at some places, while the climate remains extremely cold at other places. While there is excessive rainfall at some places, other places experience extreme dryness. So, irrigation is needed in India.

2)Irregular and uncertain monsoon: India is a land of monsoon. But monsoon is irregular and erratic in nature. Sometimes it comes easily and brings heavy rainfall, but sometimes it come late and brings inadequate rainfall. Further, there is irregularity in the distribution of rainfall throughout the year. The irrigation system helps the farmers to have less dependency on rain-water. During the months of inadequate rainfall, the crops are supplied water through irrigation systems.

3)Agriculture based economy: Indian economy is based on agriculture. A large portion of Indian population depends on agriculture. Without irrigation agriculture is not possible in dry areas or during the months of inadequate 


\section{International Journal of Science and Research (IJSR) \\ ISSN (Online): 2319-7064}

Index Copernicus Value (2013): 6.14 | Impact Factor (2014): 5.611

rainfall. Naturally, for the agricultural activities across diverse regions, there is a need for proper irrigation system.

4) Winter crop: India is a vast country with fertile plain lands suitable for Rabi as well Kharif crops. But there is no rainfall during winter months in several places. Without irrigation production of Rabi crops along with other crops is not possible. Rabi crops are grown during the long dry spell of winter season. This is possible mainly due to advanced irrigation facilities.

5)High breed seeds: At present because of high-breed seeds, crops can be produced at any seasons. But the production of crops is totally depending on water. The introduction of advanced irrigation system have enabled the farmers to produce crops even on during dry season.

6) Soil Character: In many places, the soils have less waterretaining capacities.

7)Jute and Paddy: Irrigation is needed for growing some thirsty plants like jute and paddy.

Hence, irrigation is of utmost importance for a agriculture based country like India. To feed a population of over one billion people, there is a need for production of crops round the year. But for this purpose, irrigation water is needed. So for the production of food crops and cash crops, irrigation is a must.

\subsection{Need}

The artificial way of supplying water to the agricultural land at the right moment in an appropriate volume for the proper growth of the plants in order to get the maximum yields of cultivation is technically called irrigation. But irrigation also includes clearing away of excess water from the agricultural land.

There is a great necessity of irrigation in Indian agriculture. India has a great diversity and variety of climate and weather conditions. These conditions range from extreme of heat to extreme of cold and from extreme dryness to excessive rainfall. Due to some reasons irrigation is needed in Indian agriculture.

- Uncertainty of Monsoon rainfall both in time and place. Irregularity in distribution of rainfall throughout the year.

- Excessive rainfall causing flood.

- Draught is an annual event in some areas.

- India is a land of Rabi Crops. But there is not rainfall in winter months.

- Some soils need more water.

- Introduction of H.Y.V seeds and multiple cropping need water throughout the year.

\subsection{Types}

The types of Irrigation mainly practiced in India are:

- Tanks

(a) Sichhni, (b) Donga

- Well

(a) Dug Well, (b) Tube Well: (i) Shallow. (ii) Deep.

- Canal

(a) Perennial, (b) Non-Perennial

\section{Literature Review}

After the research in the agricultural field, researchers found that the yield of agriculture goes on decreasing day by day. Use of technology in the field of agriculture plays important role in increasing the production as well as in reducing the extra man power efforts, water requirement and fertilizer requirement.

Model includes soil moisture, temperature and pressure sensors to monitor the irrigation operations. Specifically, we take into account the case where a system malfunction occurs, as when the pipes burst or the emitters block. Also, we differentiate two main traffic levels for the information transmitted by the WSAN, and we use an adequate prioritybased routing protocol to achieve high QoS performance. Simulations conducted over the NS-2 simulator show promising results in terms of delay and Packet Delivery Ratio (PDR), mainly for priority traffic [1].

System has a distributed wireless network of soil-moisture \& temperature sensors placed in root zone of plants. Gateway unit handles sensor information, triggers actuators, and transmits data to a web application. An algorithm was developed with threshold values of sensors that was programmed into a microcontroller-based gateway to control water quantity [2].

Small embedded system device (ESD) which takes care of a whole irrigation process. The PIC18F4550 microcontroller interfaced with GSM module works as a brain and several sensors like temperature, level and rain works as eyes of this ESD. If and only if eyes of the ESD sees all parameters are within a safe range, the PIC18F4550 starts irrigation process by starting the irrigation pump. The farmer gets time to time feedback from ESD through SMS about the action that has taken place by PIC18F4550 [3].

The commands from the user are processed at raspberry pi using python programming language. Arduino microcontrollers are used to receive the on/off commands from the rasperry pi using zigbee protocol. Star zigbee topology serves as backbone for the communication between raspberry pi and end devices. Raspberry pi acts a central coordinator and end devices act as various routers [4].

In the research field of wireless sensor network power efficient time is major issue which can be overcome by using ZigBee technology. The main idea is to understand how data travels through wireless medium transmission using WSN and monitoring system. Design of an irrigation system which is automated by using controllable parameter such as temperature, soil moisture and air humidity because they are the important factors to be controlled in PA(Precision Agriculture) [5].

It proposes a technique of real-time spatial interpolation using nearby ASW to predict real-time local weather parameter. Also proposes a correction technique by using sparse WSN with soil moisture sensor installed in it [6].

Wireless irrigation system for a smart home garden that can be integrated with existing smart home control systems. The 


\section{International Journal of Science and Research (IJSR) \\ ISSN (Online): 2319-7064}

Index Copernicus Value (2013): 6.14 | Impact Factor (2014): 5.611

system consists of slave nodes and a master station each of which is equipped with a wireless microcontroller. Each slave node is equipped with a temperature sensor, a soilmoister sensor, a water valve, a microcontroller and a zigbee transceiver. The slave microcontroller reads and frames the surrounding temperature of the garden's grass and trees along with soil moisture. Then, the frame is forwarded to the master station via a zig bee ad-hoc network. The master station has an embedded fuzzy logic irrigation algorithm to water the grass and trees based on a set of rules. A home web-server is interfaced with the master station for remote access monitoring and operation [7].

The system consists of distributed wireless sensor network of soil moisture, and temperature sensors placed in the crop field. To handle the sensor information Zig bee protocol used and control the water quantity programming using an algorithm with threshold values of the sensors to a microcontroller for irrigation system. [8]. An irrigation control system based on an FPGA is presented. Using RS232 interface to communicate with a host computer, the system receives irrigation commands from the decision making system and controls automatically the irrigation of 16 greenhouses or pieces of field. A control panel is designed in the system to make it easier for users to manage and intervene the irrigating. There are 16 LEDs indicating the status of water shortage. A small keyboard is used for users to input commands and 12 Seven-segment displays are used for displaying the date and time or user inputs. With many I/O pins, flexibility, easy debugging and all the control logic supported, the control system based on an FPGA represents a new trend in hardware application design [9].

Application of a wireless sensor network for low-cost wireless controlled irrigation solution and real time monitoring of water content of soil. Data acquisition is performed by using solar powered wireless acquisition stations for the purpose of control of valves for irrigation. The designed system has 3 units namely: base station unit (BSU), valve unit (VU) and sensor unit (SU). The obtained irrigation system not only prevents the moisture stress of trees and salification, but also provides an efficient use of fresh water resource [10].

\section{Proposed Block Diagram}

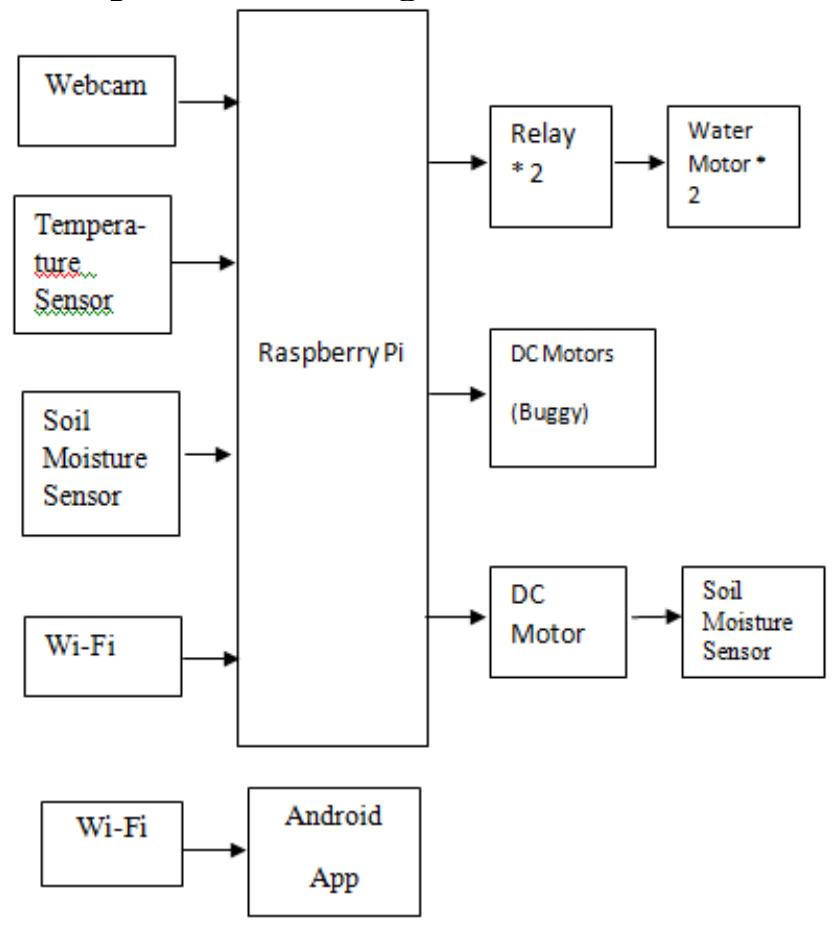

Figure 1: Block Diagram.

\section{Raspberry Pi:}

The Raspberry Pi is a low cost, credit-card sized computer. Its capable of doing everything you'd expect a desktop computer to do, from browsing the internet and playing high-definition video, making spreadsheets, and playing games. There are different models of Raspberry Pi from Raspberry Pi 0 to Raspberry Pi 3. In this project we are using Raspberry Pi Model 1 B+.

\section{Motor:}

A Motor is a device which converts electrical power into mechanical rotation using the principle of electromagnetism. Electro-magnetism: A wire wound on a ferrite core carrying electric current generates a magnetic field; this principle is called Electro-magnetism

DC motor:

DC motor in simple words is a device that converts direct current (electrical energy) into mechanical energy.

\section{Relay}

The relay module is an electrically operated switch that allows you to turn on or off a circuit using voltage and/or current much higher than a microcontroller could handle.

\section{Wi-fi dongle}

It makes Internet of Things devices cable free.

Specifications:

- Weight: $2.17 \mathrm{~g}$

- Wireless Standards: IEEE 802.11n (draft), IEEE 802.11g, IEEE $802.11 \mathrm{~b}$

- Host Interface: High speed USB2.0/1.1 interface

- Data Rate: $802.11 \mathrm{n}$ : up to $150 \mathrm{Mbps}$ (downlink) and up to 150Mbps (uplink)

Volume 5 Issue 6, June 2016 www.ijsr.net 


\section{International Journal of Science and Research (IJSR) \\ ISSN (Online): 2319-7064}

Index Copernicus Value (2013): 6.14 | Impact Factor (2014): 5.611

- Frequency Band: $2.4 \mathrm{GHz}$ ISM (Industrial Scientific Medical) Band

\section{Webcam:}

A webcam is a video camera that feeds or streams its image in real time to or through a computer to computer network. When "captured" by the computer, the video stream may be saved, viewed or sent on to other networks via systems such as the internet, and email as an attachment.

\section{Analog-to-Digital Converter (ADC):}

The MCP3204 12-bit Analog-to-Digital Converter (ADC) combines high performance and low power consumption in a small package, making it ideal for embedded control applications. The MCP3204 features a successive approximation register (SAR) architecture and an industrystandard SPITM serial interface, allowing 12-bit ADC capability to be added to any PICmicro® microcontroller.

\section{Soil moisture Sensor:}

Soil moisture sensor includes comparator (LM393) which converts analog data to discrete.

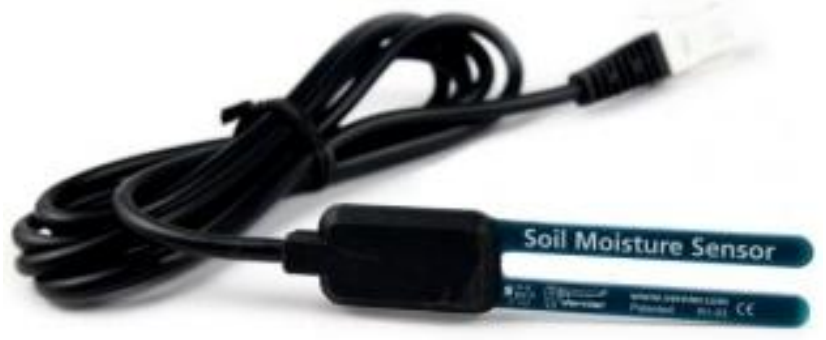

Figure 2: Soil Mositure Sensor

Two soil probes consist of two thin copper wires each of 5 $\mathrm{cm}$ length which can be immersed into the soil under test. The circuit gives a voltage output corresponding to the conductivity of soil. The soil between the probes acts as a variable resistance whose value depends upon moisture content in soil. The resistance across soil probes can vary from infinity (for completely dry soil) to a very little resistance (for $100 \%$ moisture in soil)

\section{Temperature Sensor(LM35):}

The LM35 series are precision integrated-circuit temperature devices with an output voltage linearly-proportional to the Centigrade temperature. The LM35 device has an advantage over linear temperature sensors calibrated in Kelvin, as the user is not required to subtract a large constant voltage from the output to obtain convenient Centigrade scaling.

\section{L293D:}

L293D is a dual H-bridge motor driver integrated circuit (IC). Motor drivers act as current amplifiers since they take a low-current control signal and provide a higher-current signal. This higher current signal is used to drive the motors. L293D contains two inbuilt H-bridge driver circuits. In its common mode of operation, two DC motors can be driven simultaneously, both in forward and reverse direction.

\section{System Working}

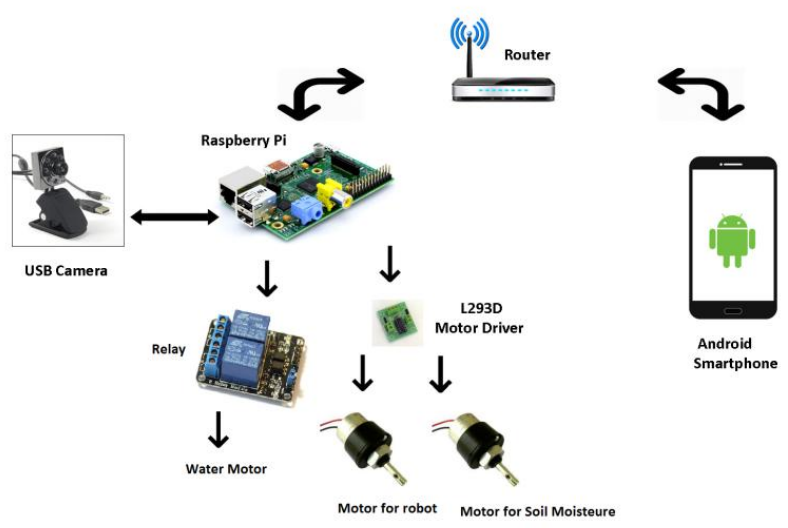

Figure 2: Pictorial representation

It consist of different types of sensing units such as Soil moisture sensor to measure water content of soil, temperature sensor to detect the temperature.

DC motor based vehicle is designed for Irrigation purpose. Webcam is interfaced to Raspberry Pi using USB port. Robot moves forward for 5 secs. It stops at the 1 st plant. Image of that plant is taken using webcam. Raspberry $\mathrm{Pi}$ performs Image Processing which gives calculates the growth of plant using height and width parameters.

If the growth of plant is improper, it will spray fertilizer on plant. If the growth of plant is proper, the soil moisture electrode is inserted in soil. It will check the value of that sensor and reat the temp value sensor. The set point for soil moisture sensor \& temperature sensor is 4000 \& 35 respectively.

If the soil moisture value is less than 4000 and temp is greater than 35 , water will be supplied for 2 secs. If the soil moisture value is less than 4000 and temp is less than 35 , water will be supplied for 1 secs. If the soil moisture value is greater than 4000 , no water supply takes place. The robot moves further for 5 secs and the same process repeats for remaining plants. The Raspberry $\mathrm{Pi}$ will send all the Information to the server using $\mathrm{Wi}-\mathrm{Fi}$. We are designing a Android App based server. The android app has a GUI which will show all the data to user.

\subsection{Data flow diagram}

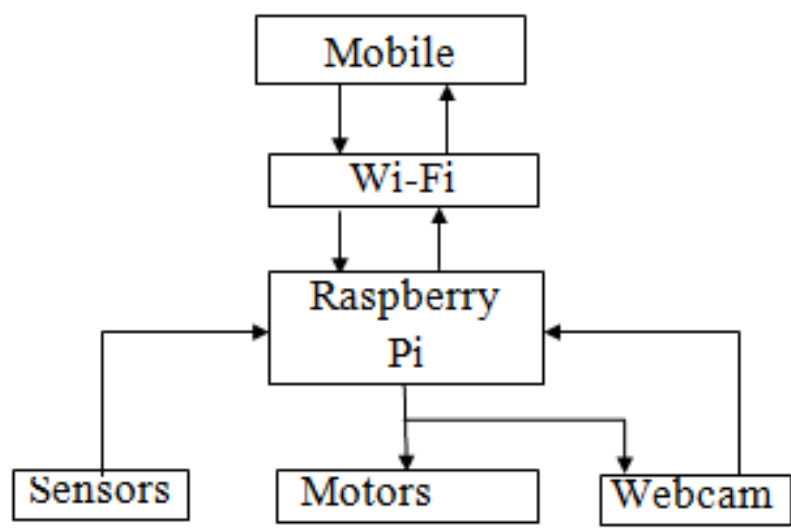

Figure 3: Data flow diagram 


\section{International Journal of Science and Research (IJSR) \\ ISSN (Online): 2319-7064}

Index Copernicus Value (2013): 6.14 | Impact Factor (2014): 5.611

\subsection{Flow Chart:}

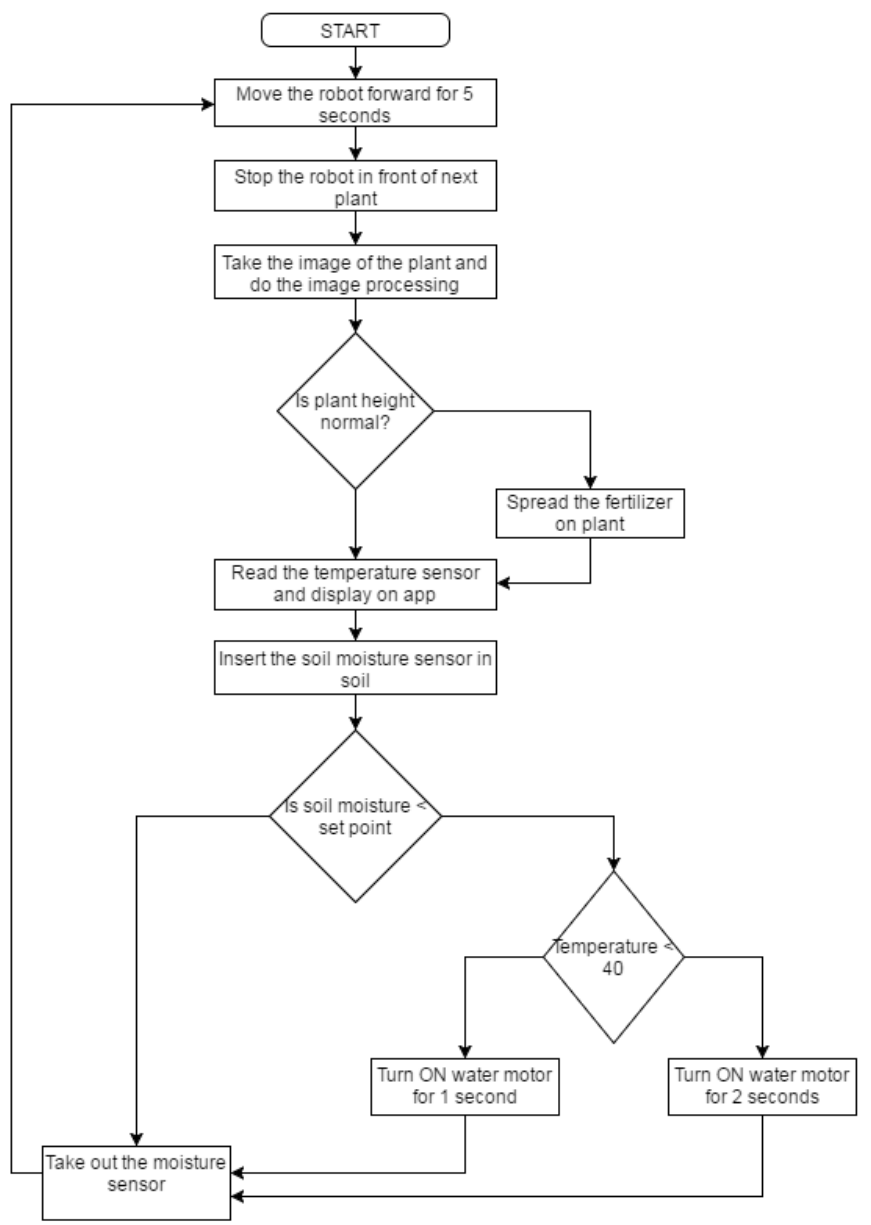

Figure 4: Flow Chart

\section{Software Description}

\subsection{Raspbian Operating System}

Raspbian is a free operating system based on Debian optimized for the Raspberry Pi hardware. It is based on the ARM Debian 7 'Wheezy' architecture port originally designed for ARMv7 and later processors (with Jazelle RCT/ThumbEE, VFPv3, and NEON SIMD extensions), compiled for the more limited ARMv6 instruction set of the Raspberry Pi.

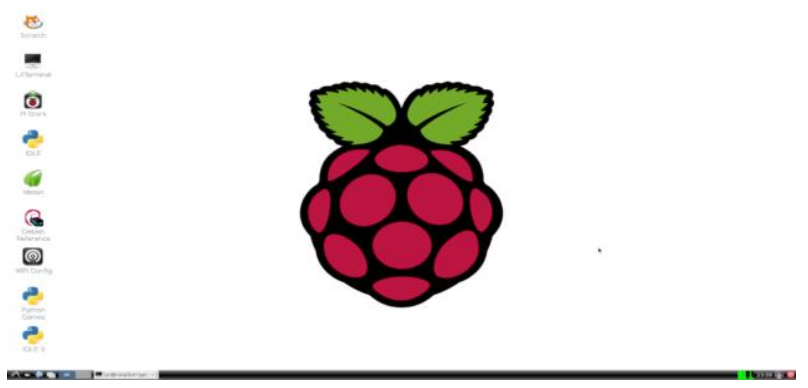

\subsection{Basics For Android:}

Basic4Android (currently known as B4A) is a rapid application development tool for native Android applications, developed and marketed by Anywhere
Software Ltd. B4A is an alternative to programming with Java and the Android SDK.

B4A includes a visual designer that simplifies the process of building user interfaces that target phones and tablets with different screen sizes. Compiled programs can be tested in AVD Manager emulators or on real Android devices using Android Debug Bridge and B4A Bridge.

The language itself is similar to Visual Basic and Visual Basic .Net though it is adapted to the native Android environment. B4A is an object-based and event-driven language.

B4A generates standard signed Android applications which can be uploaded to app stores like Google Play, Samsung Apps and Amazon Appstore. There are no special dependencies or runtime frameworks required.

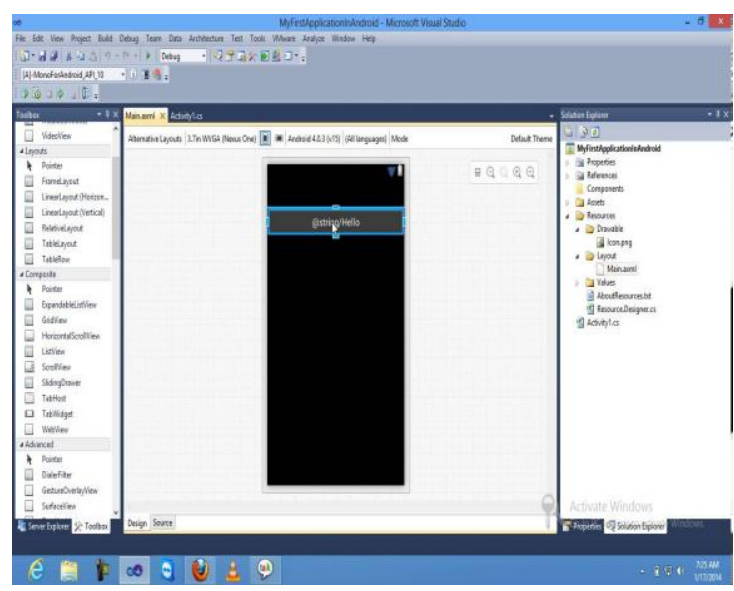

\subsection{Languages Used}

\subsubsection{Python}

Python, C, C++, Java, Scratch, and Ruby all come installed by default on the Raspberry Pi. The Pi in Raspberry Pi even comes from the Python programming language; the very idea of programming is reflected into the name of device. Python is a widely used high-level, general-purpose, interpreted, dynamic programming language. Its design philosophy emphasizes code readability, and its syntax allows programmers to express concepts in fewer lines of code than would be possible in languages such as $\mathrm{C}++$ or Java. The language provides constructs intended to enable clear programs on both a small and large scale.

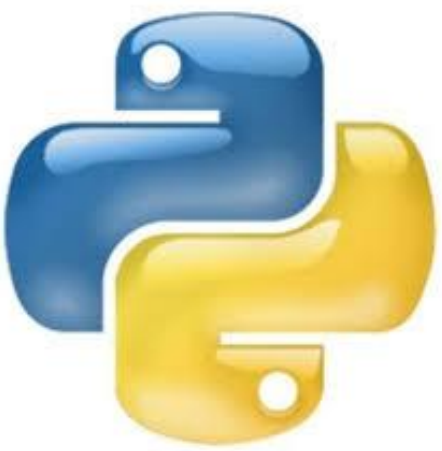




\section{International Journal of Science and Research (IJSR) \\ ISSN (Online): 2319-7064}

Index Copernicus Value (2013): 6.14 | Impact Factor (2014): 5.611

\subsubsection{Java}

Java is a programming language first released by Sun Microsystems back in 1995. It can be found on many different types of devices from smartphones, to mainframe computers. Java doesn't compile to native processor code but rather it relies on a "virtual machine" which understands an intermediate format called Java bytecode. Each platform that runs Java needs a virtual machine (VM) implementation. On Android the original VM is called Dalvik. The job of these virtual machines is to interpret the byte code, which is really just a set of instructions similar to the machine code found in CPUs, and executes the program on the processor.

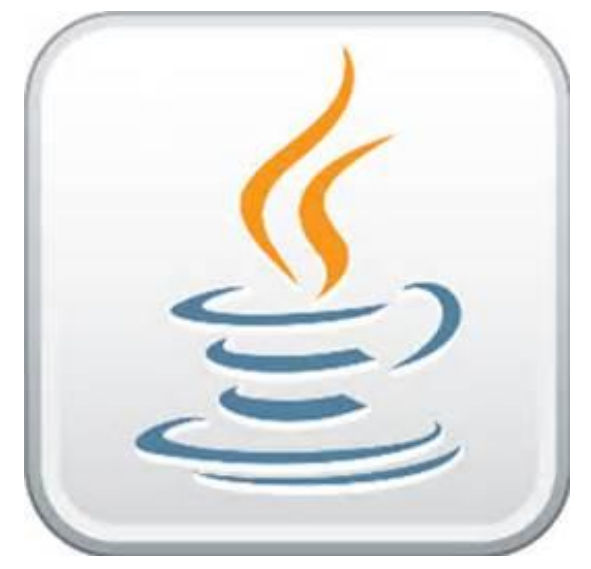

\section{Cost Analysis}

\begin{tabular}{|l|l|l|l|}
\hline Component & Unit cost & $\begin{array}{l}\text { Quantit } \\
\text { y }\end{array}$ & Total cost \\
\hline Raspberry Pi & Rs. 2500 & 1 & Rs. 2500 \\
\hline Wi-Fi dongle & Rs. 150 & 1 & Rs. 150 \\
\hline Webcam & Rs. 300 & 1 & Rs. 300 \\
\hline DC Motors & Rs. 160 & 5 & Rs. 800 \\
\hline ADC & Rs. 190 & 1 & Rs. 190 \\
\hline Relay circuit & Rs. 400 & 2 & Rs. 800 \\
\hline Sensors & Rs. 150 & 2 & Rs. 300 \\
\hline Motor Driver IC & Rs. 55 & 2 & Rs. 110 \\
\hline Other components & & & Rs. 1000 \\
\hline Total cost & & Rs. 6150 \\
\hline
\end{tabular}

\section{Results}

\subsection{Hardware Part}

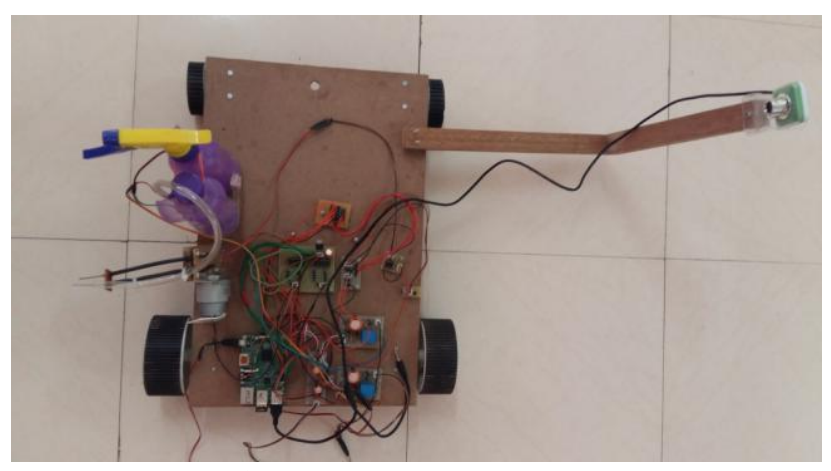

Figure 5: DC based vehicle

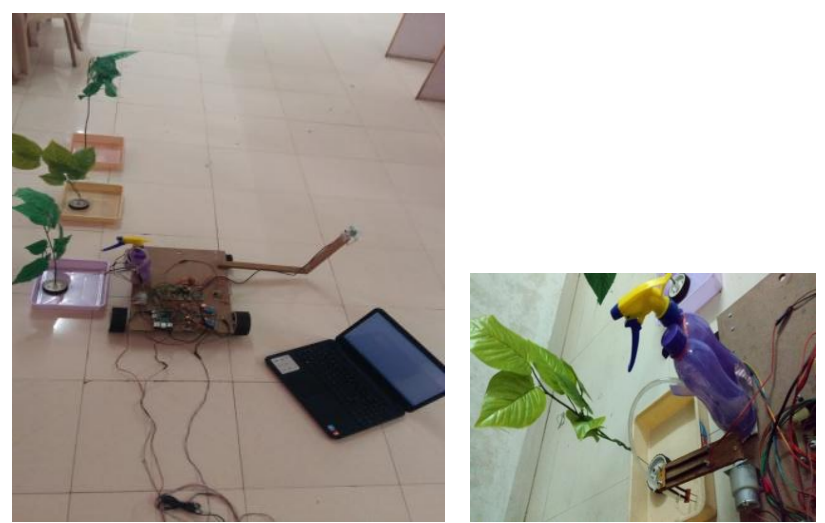

Figure 6: Project Setup Figure 7: Water supply to plant

\subsection{Software Part}

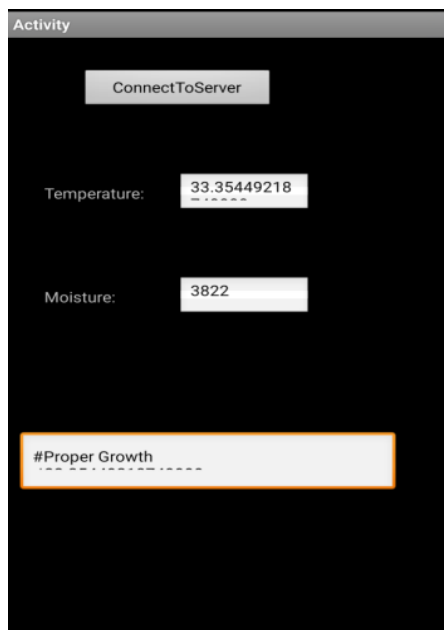

Figure 8: App Design

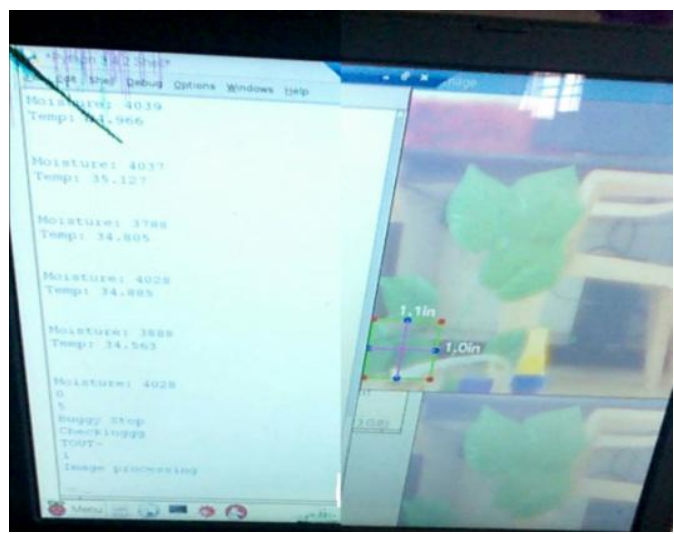

Figure 9: Plant growth detection using Image Processing

\section{Advantages}

- Moisture within the root zone can be maintained at field capacity.

- Water distribution is highly uniform, controlled by output of each nozzle.

- Labour cost is less than other irrigation methods.

- Fertigation can easily be included with minimal waste of fertilizers. 


\section{International Journal of Science and Research (IJSR) \\ ISSN (Online): 2319-7064}

Index Copernicus Value (2013): 6.14 | Impact Factor (2014): 5.611

\section{Conclusion}

- Using this system, one can save manpower, water to improve production and ultimately increase profit.

- The automated irrigation system is feasible and cost effective for optimizing water resources for agricultural production.

- The system would provide feedback control system which will monitor and control all the activities of plant growth and irrigation system efficiently.

\section{Acknowledgment}

The authors would like to thank the Department of Electronics \& Telecommunication Engineering, JSPM's J.S.C.O.E., Hadapsar for providing resources.

\section{References}

[1] I. Bennis, H. Fouchal, O. Zytoune, D. Aboutajdine, "Drip Irrigation System using Wireless Sensor Networks" Proceedings of the Federated Conference on Computer Science and Information Systems, ACSIS, Vol. 5, 2015.

[2] Joaquín Gutiérrez, Juan Francisco Villa-Medina, Alejandra Nieto-Garibay, and Miguel Ángel PortaGándara, "Automated Irrigation System Using a Wireless Sensor Network and GPRS Module," IEEE TRANSACTIONS ON INSTRUMENTATION AND MEASUREMENT, VOL. 63, NO. 1, JANUARY 2014.

[3] Sangamesh Malge, Kalyani Bhole, "Novel, Low cost Remotely operated smart Irrigation system" 2015 International Conference on Industrial Instrumentation and Control (ICIC) College of Engineering Pune, India. May 28-30, 2015

[4] Nikhil Agrawal, Smita Singhal, "Smart Drip Irrigation System using Raspberry pi and Arduino" International Conference on Computing, Communication and Automation (ICCCA2015)

[5] Pravina B. Chikankar, Deepak Mehetre, Soumitra Das, "An Automatic Irrigation System using ZigBee in Wireless Sensor Network," 2015 International Conference on Pervasive Computing (ICPC)

[6] HemaN., Krishna Kant, "Local Weather Interpolation Using Remote AWSData with Error Corrections Using Sparse WSN forAutomated Irrigation for Indian Farming", 978-1-4799-5173-4/14/\$31.00 @2014 IEEE.

[7] A. R. AI-Ali, Murad Qasaimeh, Mamoun AI-Mardinia, Suresh Radder and I. A. Zualkernan, "ZigBee-Based Irrigation System for Home Gardens" Department of Computer Science and Engineering, American University of Sharjah, UAE 978-1-4799-65328/15/\$31.00 @20 15 IEEE

[8] G. Nisha , J.Megala, Velammal institute a/technology ,Chennai,India, "WIRELESS SENSOR NETWORK BASED AUTOMATED IRRIGATION AND CROP FIELD MONITORING SYSTEM, 2014 Sixth International Conference on Advanced Computing (ICoAC) 978-1-4 799-8159- 5114/\$31.00@20 14 IEEE
[9] Zhiyong Lai, Yongli Dai, "An Irrigation Control System Based On An FPGA" 2012 Second International Conference on Instrumentation \& Measurement, Computer, Communication and Control.

[10]Mahir Dursun and Semih Ozden, "A wireless application of drip irrigation automation supported by soil moisture sensors" Scientific Research and Essays Vol. 6(7), pp. 1573-1582, 4 April, 2011. 